\title{
3D Correlative Analytical STEM at the Atomic Scale
}

\author{
David C. Bell $^{*}$, Richard Schillinger ${ }^{* *}$ and Dimitry Kolmykov ${ }^{* *}$ \\ *School of Engineering and Applied Sciences, and Center for Nanoscale Systems, \\ Harvard University, Cambridge, Massachusetts 02138 \\ **Carl Zeiss NTS GmbH, Carl Zeiss SMT, Oberkochen Germany
}

Requirement of brighter emission with less energy spread [1] as well as probe aberration correction has been a corner stone of current high-end analytical electron microscopy, we can easily image atoms on substrates as in figure 1. The combination of a probe Cs correction and an electron source monochromator yields a further step along the path to an ideal microscope. Microscopy applications that especially depend on energy resolution such as spectroscopy and advanced contrast mechanisms such as "atomic scale" energy filtered imaging can be further enhanced by the addition of monochromator. Recent work has focused on improvements to STEM technology for the applications of atomic resolution EELS and imaging, such as enhancement of collection optics of the spectrometer entrance aperture and minimization the elastic scattering artifacts $[2,3]$. The new generation of Omega in-column energy filter features second order correction and an acceptance angle of $160 \mathrm{mrad}$.

The most important fundamental concept for the collection of X-rays using XEDS system is geometry, the solid angle of the detector with respect to the sample, the collection efficiency of the detector roughly scaling with solid angle. The limited space around the microscope pole piece in a STEM make it a difficult task to improve solid angle and still retain imaging performance. By using an old idea in this new setting of an aberration corrected instrument we have increased solid angle by using twin detectors on a monochromated, Cs probe corrected column. The two detectors are mounted exactly 180 degrees with respect to each other and as close to the sample as practically possible. This allows quick acquisition time without sacrificing resolution or $\mathrm{S} / \mathrm{N}$. In this configuration the sample maintains the correct perspective relative to (HA)ADF and energy-loss spectroscopy analysis. Using a Fischione Model 2050 on-axis Tomography Holder allows high angle rotation without shadowing or substrate background signals. This system brings us to the realization of 3D atomic scale XEDS imaging. Fig. 2 and 3 show examples of ADF imaging and Pseudo 3D XEDS mapping (fig. 2) and line profile (fig. 3) on multi-component nanowires. The use of 180 degree offset detectors produces parallax of signal detection that has had the surprising advantage of simplifying the analysis of the tilted data sets, approaches to XEDS tilt series analysis will be discussed.

A single analytical platform featuring STEM probe Cs corrector, monochromator, in-column energy filter and twin x-ray detection sensitivity serves an exceptional foundation for simultaneous signal collection and an approach towards the realization of simultaneous atomic-column EELS and 3D XEDS analysis.

\section{References}

[1] P.W. Hawkes and J.C.H. Spence Science of Microscopy Springer (2007)

[2] D.A. Muller et al, Science 319:1073-1076. (2008).

[3] S.J. Pennycook, et. al., J. Electron Microsc. (Tokyo), January (2009) 


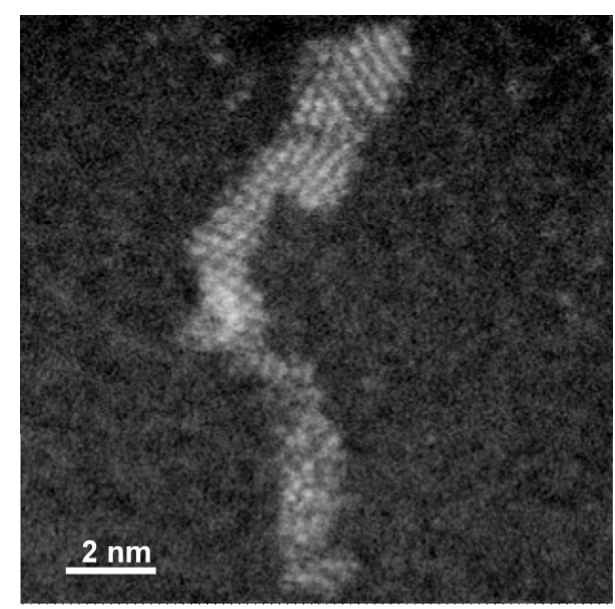

Figure 1. Repeating A. Crewe's groundbreaking experiment - Thorium atoms imaged "today" on an aberration corrected STEM (Zeiss Libra 200 MC Cs STEM).

Fig. 2. Pseudo 3D STEM imaging and 180 Degree - EDS mapping of singlecrystalline kinked semiconductor nanowire superstructures (sample courtesy of C. Lieber, Harvard University). Data set collected using twin XEDS detectors.
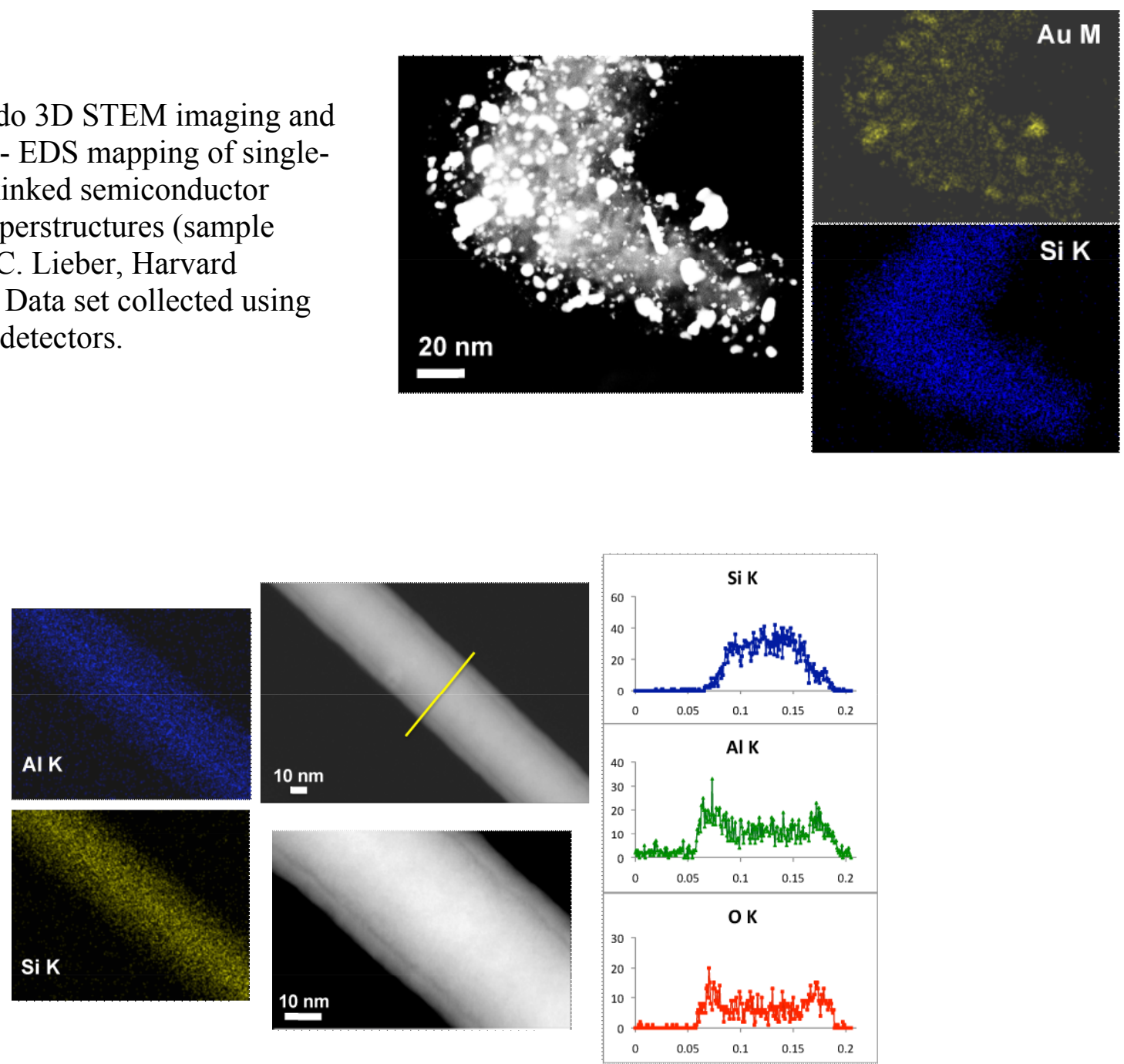

Fig. 3. STEM imaging and EDS (maps and line scan) of Si nanowire with $\mathrm{AlO}_{\mathrm{x}}$ coating. Data combined from twin XEDS detectors. 